\title{
COMPLETELY CONTINUOUS MOVEMENTS IN TOPOLOGICAL VECTOR SPACES
}

\author{
by J. H. MICHAEL \\ (Received 4th June, 1957)
}

1. Introduction. Let $A$ be a closed subset of a topological space $X$ and $f$ a continuous mapping of $A$ into $X$ with the following two properties :

1.1. $f\{\operatorname{Fr}(A)\}$ and $f\{\operatorname{Int}(A)\}$ are disjoint.

1.2. The mapping $f_{*}=f \mid \operatorname{Fr}(A)$ is $1-1$.

It is proved in [5], that if $X$ is the euclidean $n$-sphere $S^{n}=\left\{x ; x \in R^{n+1}\right.$ and $\left.\|x\|=1\right\}$, then

1.3. $f\{\operatorname{Fr}(A)\}=\operatorname{Fr}\{f(A)\}$.

[Hence $f\{\operatorname{Int}(A)\}=\operatorname{Int}\{f(A)\}]$.

The purpose of the present paper is to prove (Theorem 4.4.) that 1.3 is true when $X$ is a real convex topological vector space with a Hausdorff topology and $f$ satisfies the additional requirement of being a completely continuous movement. The proof of this theorem makes use of the degree of completely continuous movements.

The writer is indebted to Dr A. P. Robertson and Dr Wendy Robertson for their valuable advice.

2. Notation. We shall assume henceforth that $E$ denotes a real convex topological vector space with a Hausdorff topology. $\mathscr{U}$ is the collection of all convex symmetrical open neighbourhoods of the origin. In the usual way, a mapping $f$ of a subset $A$ of $E$ into $E$ is defined to be completely continuous on $A$ if it is continuous and there exists a compact subset $K$ of $E$ with $f(A) \subseteq K$. Following Nagumo [6], we define a completely continuous movement of a subset $A$ of $E$ to be a mapping $f$ of $A$ into $E$, such that the function

$$
\phi(x) \equiv f(x)-x
$$

is completely continuous on $A$. Set complementation is denoted by $\sim$; i.e., if $B \subseteq A$, then $A \sim B$ denotes the complement of $B$ in $A$.

3. The degree of a completely continuous movement.

3.1. We first of all observe that completely continuous movements have the following properties. These are proved in [6].

3.1.1. If $A$ is a closed subset of $E$ and $f$ is a completely continuous movement of $A$, then $f(A)$ is closed.

3.1.2. If $f, g$ are completely continuous movements of $A, f(A)$, respectively, then $g f$ is $a$ completely continuous movement of $A$.

3.1.3. If $f$ is a 1-1 completely continuous movement of $A$ and $A$ is closed, then $f^{-1}$ is a completely continuous movement of $f(A)$.

3.2. Consider the triple $(f, A, b)$, where $A$ is a subset of $E, f$ is a completely continuous movement of $\operatorname{Fr}(A)$ and $b$ is a point of $E \sim f\{\operatorname{Fr}(A)\}$. With each such triple there is associated an integer

$$
d(f, A, b)
$$


called its degree. $\dagger$ This degree has the following properties.

3.2.1. If $f$ is the identity mapping of $\operatorname{Fr}(A)$, then

$$
\begin{aligned}
d(f, A, x) & =1 & & \text { when } x \in \operatorname{Int}(A), \\
& =0 & & \text { when } x \in E \sim \bar{A} .
\end{aligned}
$$

3.2.2. If $f$ is a completely continuous movement of $\bar{A}$ and $b \notin f(\bar{A})$, then

$$
d(f, A, b)=0 .
$$

3.2.3. Let $\mathscr{G}$ be a collection of mutually disjoint open subsets of $\operatorname{Int}(A)$. Put

$$
U=\underset{G \in \mathscr{G}}{\mathrm{U}} G
$$

If $f$ is a completely continuous movement of $\bar{A} \sim U$ and $b \in E \sim f(\bar{A} \sim U)$, then $d(f, G, b)=0$ for all but a finite number of $G \in \mathscr{G}$ and

$$
d(f, A, b)=\sum_{G \in \mathscr{G}} d(f, G, b) .
$$

3.2.4. If $f$ is a completely continuous movement of $\operatorname{Fr}(A)$ and $b_{1}, b_{2}$ are points of the same component of $E \sim f\{\operatorname{Fr}(A)\}$, then

$$
d\left(f, A, b_{1}\right)=d\left(f, A, b_{2}\right) .
$$

3.2.5. If $f$ is a completely continuous movement of $\operatorname{Fr}(A), b \in E \sim f\{\operatorname{Fr}(A)\}, U \in \mathscr{U}$ and $U$ is such that $b+U$ does not intersect $f\{\operatorname{Fr}(A)\}$ and if $f_{1}$ is a completely continuous movement of $\operatorname{Fr}(A)$ such that

for all $x \in \operatorname{Fr}(A)$, then

$$
f(x)-f_{1}(x) \in U
$$

$$
d(f, A, b)=d\left(f_{1}, A, b\right) .
$$

3.2.6. Let $B$ be a second subset of $E, f$ and $g$ be completely continuous movements of $\bar{A}$ and $\bar{B}$ such that $f(\bar{A}) \subseteq \bar{B}$, and $b$ be a point of $E \sim g[\operatorname{Fr}(B) \cup f\{\operatorname{Fr}(A)\}]$ such that $d(f, A, y)$ is constant for $y \in g^{-1}(b)$. Then

$$
\begin{aligned}
d(g f, A, b) & =d(g, B, b) . d\left\{f, A, g^{-1}(b)\right\} \text { if } g^{-1}(b) \neq \emptyset, \\
& =0 \text { if } g^{-1}(b)=\emptyset .
\end{aligned}
$$

[Here $d\left\{f, A, g^{-1}(b)\right\}$ denotes the constant value of $d(f, A, y)$ for $\left.y \in g^{-1}(b)\right]$.

Further properties of the degree are given by Theorems 3.2.7 and 3.2.9, which appear below. Theorem 3.2.9 is not new (it is used by Leray in [3], for example), but since the proof does not appear to be readily available in the literature, the theorem is proved here.

3.2.7. Theorem. Let $A$ be a subset of $E, f$ be a completely continuous movement of $\operatorname{Fr}(A)$ and $b \in E \sim f\{\operatorname{Fr}(A)\}$. Let $K$ be a compact subset of $E$ such that

$$
f(x)-x \in K
$$

for all $x \in \operatorname{Fr}(A)$. If $F$ is a linear manifold of $E$ which contains $b$ and $K$, then

$$
d(f, A, b)=d(f, F \cap A, b) \text { in } F .
$$

Proof. Choose $U \in \mathscr{U}$ such that $b+U$ does not intersect $f\{\operatorname{Fr}(A)\} . \quad K$ is evidently compact in $F$; hence, by Theorem 2 of [6], there exist a finite dimensional linear manifold $G$ of $F$ containing $b$, and a continuous mapping $\phi$ of $K$ into $G$ such that

$$
\phi(x)-x \in U,
$$

$\dagger$ For the definition and properties of the degree, see [3] and [6]. Actually, in [3] and [6] the degree is defined for open $A$. However, it can easily be defined for arbitrary $A$ by putting

$$
d(f, A, b)=d\{f, \operatorname{Int}(A), b\} \text {. }
$$


for all $x \in K$. Put

for all $x \in \operatorname{Fr}(A)$. Since

$$
f_{1}(x)=\phi\{f(x)-x\}+x
$$

$$
f_{1}(x)-x=\phi\{f(x)-x\} \in \phi(K),
$$

and $\phi(K)$ is compact, $f_{1}(x)$ is a completely continuous movement of $\operatorname{Fr}(A)$. Also

$$
f(x)-f_{1}(x)=-[\phi\{f(x)-x\}-\{f(x)-x\}]
$$

hence

$$
f(x)-f_{1}(x) \in U
$$

for all $x \in \operatorname{Fr}(A)$. Furthermore, it follows from (1) that

$$
f_{1}(x)-x \in G
$$

for all $x \in \operatorname{Fr}(A)$. Now, by (2) and 3.2.5,

and, by 3.2 .3 , this is

$$
d(f, A, b)=d\left(f_{1}, A, b\right),
$$

which, by (3) and [3], equals

$$
d\left(f_{1}, \text { Interior of } A \text { in } E, b\right),
$$

$\left\{d f_{1}, G \cap(\right.$ Interior of $A$ in $\left.E), b\right\}=d\left\{f_{1}, F \cap(\right.$ Interior of $A$ in $\left.E), b\right\} ;$

hence, by 3.2 .3 ,

$$
d(f, A, b)=d\left(f_{1}, F \cap A, b\right),
$$

and, since (Frontier of $(F \cap A)$ in $F) \subseteq($ Frontier of $A$ in $E) \cap F$, we have, by (2) and 3.2.5,

$$
d(f, A, b)=d(f, F \cap A, b) .
$$

3.2.8. Lemma. If $F$ is a linear manifold of $E$ and $K$ is a compact subset of $F$ which spans $F$, then the relative topology of $F$ is normal.

Proof. The relative topology of $F$ is regular and Lemma 1 on p. 113 of [1] shows that a regular Lindelöf space is normal. Hence we have only to prove that $F$ is a Lindelöf space, i.e. that each covering of $F$ by open sets of $F$ has a countable subcovering.

To this end, let $\mathscr{V}$ be an open covering of $F$. For each positive integer $n$, let $K_{n}$ be the set of all points

$$
\lambda_{1} x_{1}+\ldots+\lambda_{n} x_{n}
$$

of $F$, where $x_{1}, \ldots, x_{n} \in K$ and $\lambda_{1}, \ldots, \lambda_{n}$ are real numbers such that $\left|\lambda_{1}\right| \leqslant n, \ldots,\left|\lambda_{n}\right| \leqslant n$. If $J_{n}$ denotes the closed interval $[-n, n]$, then $K_{n}$ is a continuous image of the compact space $J_{n} \times \ldots \times J_{n} \times K \times \ldots \times K$ ( $2 n$ factors) ; hence $K_{n}$ is compact. Evidently

$$
F=\bigcup_{n=1}^{\infty} K_{n} .
$$

For each $n$ we can choose a finite subcollection $\mathscr{V}_{n}^{\prime}$ of $\mathscr{V}$ which covers $K_{n}$. Let

$$
\mathscr{V}^{\prime}=\bigcup_{n=1}^{\infty} \mathscr{V}_{n}^{\prime}
$$

$\mathscr{V}^{\prime}$ is countable, $\mathscr{V}^{\prime} \subseteq \mathscr{V}$ and $\mathscr{V}^{\prime}$ covers $F$. This completes the proof.

3.2.9. Theorem. If $A$ and $B$ are subsets of $E$ with Int $(B) \neq \emptyset, f$ is a completely continuous movement of $\bar{A}$ into $\bar{B}$ such that $f\{\operatorname{Fr}(A)\} \subseteq \operatorname{Fr}(B), g$ is a completely continuous movement of $\operatorname{Fr}(B), b \in E \sim g\{\operatorname{Fr}(B)\}$ and $d(f, A, y)$ is constant for $y \in \operatorname{Int}(B)$, then

$$
d(g f, A, b)=d(g . B, b) . d\{f, A, \operatorname{Int}(B)\} .
$$


Proof. Let $K$ and $L$ be compact subsets of $E$ such that

for all $x \in \bar{A}$ and

$$
f(x)-x \in K
$$

$$
g(x)-x \in L
$$

for all $x \in \operatorname{Fr}(B)$. Let $b_{1} \in \operatorname{Int}(B)$ and $F$ be the linear manifold spanned by the compact set $K \cup L \cup\left\{b, b_{1}\right\}$. By 3.2.7,

and

$$
d\{f, A, \text { Int }(B)\}=d\{f, F \frown A, F \frown \operatorname{Int}(B)\}
$$

$$
d(g, B, b)=d(g, F \cap B, b) .
$$

But, since $K+L \subseteq F, K+L$ is compact and, for all $x \in \operatorname{Fr}(A)$, we have

$$
g f(x)-x=\{f(x)-x\}+[g\{f(x)\}-f(x)] \epsilon K+L,
$$

it also follows from 3.2.7 that

$$
d(g f, A, b)=d(g f, F \cap A, b) .
$$

Thus, it will be sufficient to prove that

$$
d(g f, F \cap A, b)=d(g, F \cap B, b) . d\{f, F \cap A, F \cap \operatorname{Int}(B)\} .
$$

By 3.1.1, $g\{F \cap \operatorname{Fr}(B)\}$ is closed in $F$; hence there exists an open, convex, symmetrical neighbourhood $U$ of the origin in $F$ such that

$$
(b+U) \cap g\{F \cap \operatorname{Fr}(B)\}=\varnothing .
$$

By Theorem 2 of [6], we can find a finite dimensional linear manifold $F^{m}$ of $F$ and a continuous mapping $\theta$ of $L$ into $F^{m}$ such that

for all $x \in L . \quad$ Put

$$
\theta(x)-x \in U
$$

$$
\psi_{1}(x)=\theta\{g(x)-x\}
$$

for all $x \in F \cap \operatorname{Fr}(B)$. Now $\theta(L)$ is a compact subset of $F^{m}, F \cap \operatorname{Fr}(B)$ is closed in $F, \psi_{1}$ is a continuous mapping of $F \cap \operatorname{Fr}(B)$ into $\theta(L)$ and, by Lemma 3.2.8, $F$ is normal. Hence one can apply Tietze's Extension Theorem ([2], p. 28) to extend $\psi_{1}$ to a continuous mapping of $F \cap \bar{B}$ into a compact subset $L_{1}$ of $F^{m}$. Put

$$
g_{1}(x)=\psi_{1}(x)+x
$$

for all $x \in F \cap \bar{B}$. Then $g_{1}$ is a completely continuous movement of $F \cap \bar{B}$ into $F$ and for all $x \in F \cap \operatorname{Fr}(B)$ we have $g(x)-g_{1}(x)=\{g(x)-x\}-\theta\{g(x)-x\}$, and hence

$$
g(x)-g_{1}(x) \in U
$$

for all $x \in F \cap \operatorname{Fr}(B)$. Since the frontier of $F \cap B$ in $F$ is contained in $F \cap \operatorname{Fr}(B)$, it follows from (8), (9) and 3.2.5 that

$$
d(g, F \cap B, b)=d\left(g_{1}, F \cap B, b\right)
$$

Since $f\{F \cap \operatorname{Fr}(A)\} \subseteq F \cap \operatorname{Fr}(B)$, we obtain from (9)

for all $x \in F \cap \operatorname{Fr}(A)$; hence

$$
g f(x)-g_{1} f(x) \in U
$$

$$
d(g f, F \cap A, b)=d\left(g_{1} f, F \cap A, b\right) .
$$

Now it follows from (8) and (9) that $g_{1}^{-1}(b) \subseteq F \cap \operatorname{Int}(B)$; hence, by (4), $d(f, F \cap A, y)$ is constant for $y \in g_{1}^{-1}(b)$. Therefore, by 3.2.6,

$$
d\left(g_{1} f, F \cap A, b\right)=d\left(g_{1}, F \cap B, b\right) . d\{f, F \cap A, F \cap \operatorname{Int}(B)\} .
$$

Equation (7) now follows immediately from (10), (11) and (12), and the proof is complete. 
4. The main result. In this section we prove the theorem that was discussed in $\S 1$. Throughout the section $A$ denotes a closed subset of $E$ and $f$ a completely continuous movement of $A$ with the properties 1.1 and 1.2. As in 1.2, $f_{*}=f \mid \operatorname{Fr}(A)$. then

4.1. Lемма. If $Q$ is a component of $E \sim f\{\operatorname{Fr}(A)\}$ which intersects $f(A)$ and if $P=f^{-1}(Q)$,

(i) $Q$ does not intersect $f\{\operatorname{Fr}(P)\}$, and

for all $y \in Q$;

$$
d(f, P, y) \neq 0
$$

(ii) $\operatorname{Fr}(Q) \subseteq f\{\operatorname{Fr}(A)\}, f_{*}^{-1}\{\operatorname{Fr}(Q)\}$ does not intersect $P$ or $E \sim \bar{P}$ and

$$
\begin{aligned}
d\left(f_{*}^{-1}, Q, x\right) & \neq 0 \text { for } x \in P, \\
& =0 \text { for } x \in E \sim \bar{P} .
\end{aligned}
$$

Proof. $P$ and $Q$ are evidently open sets of $E$ and

$$
\operatorname{Fr}(Q) \subseteq f\{\operatorname{Fr}(A)\} \text {. }
$$

If $a$ is an arbitrary point of $\operatorname{Fr}(P)$, then $f(a) \epsilon \bar{Q}$ and $f(a) \notin Q$; for $f(a) \in Q$ would imply $a \in P$. Hence $f(a) \in \operatorname{Fr}(Q)$. Thus

$$
f\{\operatorname{Fr}(P)\} \subseteq \operatorname{Fr}(Q)
$$

Hence

Also

$$
Q \cap f\{\operatorname{Fr}(P)\}=\varnothing
$$

$$
P \cap f_{*}^{-1}\{\operatorname{Fr}(Q)\}=\emptyset
$$

for, if $a^{\prime} \in P$, then $f\left(a^{\prime}\right) \in Q$. Hence $f\left(a^{\prime}\right) \notin \operatorname{Fr}(Q)$; i.e., $a^{\prime} \notin f_{*}^{-1}\{\operatorname{Fr}(Q)\}$. Now

$$
\operatorname{Fr}(P) \subseteq \operatorname{Fr}(A) \text {. }
$$

For otherwise there would exist a point $p \in \operatorname{Fr}(P)$ with $p \in \operatorname{Int}(A)$; then, by 1.1,

$$
f(p) \notin f\{\operatorname{Fr}(A)\},
$$

which contradicts (13) and (14). By (14), 3.2.4 and Theorem 3.2.9,

$$
d\left(f_{*}^{-1} f, P, x\right)=d\left(f_{*}^{-1}, Q, x\right) . d(f, P, y)
$$

for all $x \in E \sim f_{*}^{-1}\{\operatorname{Fr}(Q)\}$ and all $y \in Q$. Therefore by (15), 1.1 and 3.2.1,

$$
\begin{aligned}
d\left(f_{*}^{-1}, Q, x\right) . d(f, P, y) & =1, \text { for } x \in P, \\
& =0, \text { for } x \in E \sim\left[\vec{P} \cup f_{*}^{-1}\{\operatorname{Fr}(Q)\}\right],
\end{aligned}
$$

for all $y \in Q$; consequently, since $P$ is not empty,

$$
d(f, P, y) \neq 0
$$

for all $y \in Q$. It now follows from (18) and 3.2.2 that $Q \subseteq f(\bar{P})$; hence, since $f(\bar{P})$ is closed, $\bar{Q} \subseteq f(\bar{P})$; therefore

$$
(E \sim \bar{P}) \frown f_{*}^{-1}\{\operatorname{Fr}(Q)\}=\varnothing .
$$

For, if $c \in f_{*}^{-1}\{\operatorname{Fr}(Q)\}$, then $f(c) \in \bar{Q}, f(c) \in f(\bar{P})$, and, since, by $(13), 1.1$ and $1.2, c$ is the only point in $f^{-1}\{f(c)\}$, we have $c \in \bar{P}$. Since $Q$ is not empty, it now follows from (17), (18) and (19) that

$$
\begin{aligned}
d\left(f_{*}^{-1}, Q, x\right) & \neq 0, \quad \text { for } x \in P, \\
=0, & \text { for } x \in E \sim \bar{P} .
\end{aligned}
$$


4.2. Lemma. $\operatorname{Fr}\{f(A)\} \subseteq f\{\operatorname{Fr}(A)\}$.

Proof. Suppose that the lemma is not true ; i.e., that there exists a point $b \in \operatorname{Fr}\{f(A)\}$ with $b \notin f\{\operatorname{Fr}(A)\}$. Let $Q$ be the component of $E \sim f\{\operatorname{Fr}(A)\}$ containing $b$ and put $P=f^{-1}(Q)$. By Lemma 4.1, $d(f, P, y) \neq 0$ for all $y \in Q$. But, since $Q$ is open and $b \in \operatorname{Fr}\{f(A)\}, Q$ must contain a point $y^{\prime}$ of $E \sim f(A)$; hence $y^{\prime} \notin f(\bar{P})$ and, by $3.2 .2, d\left(f, P, y^{\prime}\right)=0$. This is a contradiction.

4.3. Lemma. $d\left\{f_{*}^{-1}, f(A), x\right\} \neq 0$, for $x \in \operatorname{Int}(A)$,

$$
=0 \text {, for } x \in E \sim A \text {. }
$$

Proof. Let $x \in E \sim \operatorname{Fr}(A)$. Denote by $\mathscr{Q}$ the collection consisting of all those components of $E \sim f\{\operatorname{Fr}(A)\}$ that are contained in $f(A)$. By Lemma 4.2,

$$
f(A) \sim f\{\operatorname{Fr}(A)\}=\underset{Q \in \mathscr{Q}}{\cup} Q
$$

Hence, by 3.2.3,

$$
d\left\{f_{*}^{-1}, f(A), x\right\}=\sum_{Q \in \mathcal{Q}} d\left(f_{*}^{-1}, Q, x\right)
$$

(Empty sums are regarded as zero.)

Suppose that $x \in \operatorname{Int}(A)$. By 1.1 and (20), there is exactly one $Q \in \mathscr{Q}$, say $Q^{\prime}$, such that $x \in f^{-1}(Q)$. Therefore, by Lemma 4.1,

$$
\begin{aligned}
& d\left(f_{*}^{-1}, Q, x\right) \neq 0 \text {, when } Q=Q^{\prime} \text {, } \\
& =0 \text {, when } Q \neq Q^{\prime} \text {. }
\end{aligned}
$$

Hence by $(21), d\left\{f_{*}^{-1}, f(A), x\right\} \neq 0$.

If $x \in E \sim A$, there is no $Q \in \mathscr{Q}$ with $x \in f^{-1}(Q)$, so that, by $(21), d\left\{f_{*}^{-1}, f(A), x\right\}=0$.

4.4. Theorem. $f\{\operatorname{Fr}(A)\}=\operatorname{Fr}\{f(A)\}$.

Proof. Because of Lemma 4.2, we have only to prove that

$$
f\{\operatorname{Fr}(A)\} \subseteq \operatorname{Fr}\{f(A)\} \text {. }
$$

Suppose that this inequality is not true; i.e., that there exists a point $b \in f\{\operatorname{Fr}(A)\}$ with $b \notin \operatorname{Fr}\{f(A)\}$. Put $a=f_{*}^{-1}(b)$. Then $a \in \operatorname{Fr}(A)$ and $b \in \operatorname{Int}\{f(A)\}$.

(i) Let $a \in \overline{\operatorname{Int}(A)}$. Let $C$ be the component of $E \sim f_{*}^{-1}[\operatorname{Fr}\{f(A)\}]$ which contains $a$. Then $C$ is open and therefore contains a point $a^{\prime}$ of $\operatorname{Int}(A)$ and a point $a^{\prime \prime}$ of $E \sim A$. By 3.2.4,

$$
d\left\{f_{*}^{-1}, f(A), a^{\prime}\right\}=d\left\{f_{*}^{-1}, f(A), a^{\prime \prime}\right\}
$$

and this contradicts Lemma 4.3.

(ii) Let $a \notin \overline{\operatorname{Int}(A)}$. We have $b \notin f\{\overline{\operatorname{Int}(A)}\}$. Since $b \in \operatorname{Int}\{f(A)\}$, there exists a $U \in \mathscr{U}$ with $\overline{b+U}$ contained in $f(A)$ but not intersecting $f\{\overline{\operatorname{Int}(A)}\}$. Then $\overline{b+U} \subseteq f\{\operatorname{Fr}(A)\}$ and $f_{*}^{-1} \mid \overline{b+U}$ is a l-1 completely continuous movement. Therefore, by Lemma 4.2 ,

$$
\operatorname{Fr}\left\{f_{*}^{-1}(\overline{b+\bar{U}})\right\} \subseteq f_{*}^{-1}\{\operatorname{Fr}(\overline{b+\bar{U}})\},
$$

so that $a \notin \operatorname{Fr}\left\{f_{*}^{-1}(\overline{b+U})\right\}$, and hence $a \in \operatorname{Int}\left\{f_{*}^{-1}(\overline{b+U})\right\} \subseteq \operatorname{Int}(A)$. This is a contradiction.

\section{REFERENCES}

1. Kelley, J. L., General topology (New York, 1955).

2. Lefschetz, S., Algebraic topology, Amer. Math. Soc. Colloquium Publications, 27 (1942). 
3. Leray, J., La théorie des points fixes et ses applications en analyse, Rend. Sem. Mat., 15 (1955-56), 65-74.

4. Leray, J., and Schauder, J., Topologie et équations fonctionelles, Ann. Sci. École Norm. Sup., 51 (1934), 45-78.

5. Michrel, J. H., Continuous mappings of subsets of the euclidean $n$-sphere, Bull. Acad. Polon. Sci., 5 (1957), 133-137.

6. Nagumo, Mitio, Degree of mapping in convex linear topological spaces, Amer. J. Math., 73 (1951), 497-511.

\section{The University}

GLASGOW

\section{ON CERTAIN RELATIONS BETWEEN PRODUCTS OF BILATERAL HYPERGEOMETRIC SERIES}

\section{by HARISHANKER SHUKLA}

(Received 12th April, 1957)

1. Introduction. Darling [3] in 1932 and Bailey [2] in 1933 gave certain theorems on products of hypergeometric series. Again in 1948 Sears [4] used the relation which expresses the ${ }_{M} \Phi_{M-1}(x)$ series in terms of $M$ other series of the same type to derive transformations between products of both basic and ordinary hypergeometric series. In this paper I give certain general theorems on products of bilateral hypergeometric series together with some of their interesting special cases.

The following notation is used throughout the paper :

$$
\begin{aligned}
& (a ; n)=(1-a)(1-a q) \ldots\left(1-a q^{n-1}\right), \quad(a ; 0)=1,
\end{aligned}
$$

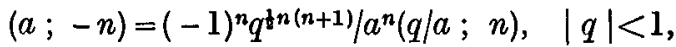

$$
\begin{aligned}
& (a)_{n}=a(a+1) \ldots(a+n-1),(a)_{0}=1, \quad(a)_{-n}=(-1)^{n} /(1-a)_{n} \text {, } \\
& { }_{r} \Psi_{r}\left[\begin{array}{l}
a_{1}, a_{2}, \ldots, a_{r} ; z \\
b_{1}, b_{2}, \ldots, b_{r}
\end{array}\right]=\sum_{n=-\infty}^{\infty} \frac{\left(a_{1} ; n\right)\left(a_{2} ; n\right) \ldots}{\left(b_{1} ; n\right)\left(b_{2} ; n\right) \ldots} \frac{\left(a_{r} ; n\right)}{\left(b_{r} ; n\right)} z^{n}, \\
& { }_{r} H_{r}\left[\begin{array}{l}
a_{1}, a_{2}, \ldots, a_{r} ; z \\
b_{1}, b_{2}, \ldots, b_{r}
\end{array}\right]=\sum_{n=-\infty}^{\infty} \frac{\left(a_{1}\right)_{n}\left(a_{2}\right)_{n} \ldots\left(a_{r}\right)_{n}}{\left(b_{1}\right)_{n}\left(b_{2}\right)_{n} \ldots\left(b_{r}\right)_{n}} z^{n}, \\
& \Pi\left[\begin{array}{c}
a_{1}, a_{2}, \ldots, a_{r} \\
b_{1}, b_{2} \ldots, b_{r}
\end{array}\right]=\prod_{n=0}^{\infty} \frac{\left(1-a_{1} q^{n}\right)\left(1-a_{2} q^{n}\right) \ldots\left(1-a_{r} q^{n}\right)}{\left(1-b_{1} q^{n}\right)\left(1-b_{2} q^{n}\right) \ldots\left(1-b_{r} q^{n}\right)}, \\
& \Gamma\left[\begin{array}{l}
a_{1}, a_{2}, \ldots, a_{r} \\
b_{1}, b_{2}, \ldots, b_{r}
\end{array}\right]=\frac{\Gamma\left(a_{1}\right) \Gamma\left(a_{2}\right) \ldots \Gamma\left(a_{r}\right)}{\Gamma\left(b_{1}\right) \Gamma\left(b_{2}\right) \ldots \Gamma\left(b_{n}\right)},
\end{aligned}
$$

and idem $(a ; b)$ means that the preceding expression is repeated with $a$ and $b$ interchanged. 